\title{
ADVANCES IN MULTI-PHYSICS AND HIGH PERFORMANCE COMPUTING IN SUPPORT OF NUCLEAR REACTOR POWER SYSTEMS MODELING AND SIMULATION
}

\author{
PAUL J. TURINSKY \\ Department of Nuclear Engineering \\ North Carolina State University \\ *Corresponding author. E-mail : turinsky@ ncsu.edu
}

Invited March 23, 2011

Received January 02, 2012

Accepted for Publication January 22, 2012

\begin{abstract}
Significant advances in computational performance have occurred over the past two decades, achieved not only by the introduction of more powerful processors but the incorporation of parallelism in computer hardware at all levels. Simultaneous with these hardware and associated system software advances have been advances in modeling physical phenomena and the numerical algorithms to allow their usage in simulation. This paper presents a review of the advances in computer performance, discusses the modeling and simulation capabilities required to address the multi-physics and multi-scale phenomena applicable to a nuclear reactor core simulator, and present examples of relevant physics simulation codes' performances on high performance computers.
\end{abstract}

KEYWORDS : High Performance Computing, Simulation, Modeling, Multi-physics, Multi-scale

\section{INTRODUCTION}

The development of nuclear energy, whether for peaceful or military application, has been highly dependent upon modeling and simulation of the physical phenomena involved. This has been necessary due to cost and infeasibility of performing experiments at the scale required that cover the total span of possible reactor conditions, including those associated with accident conditions. That is not to say that there is not a wealth of experiments that have been performed, required to complete validation of the modeling and simulation capabilities being developed.

Because of this heavy reliance on modeling and simulation, nuclear energy development has been dependent upon utilizing scientific computing facilities. Advantage has taken of both hardware and software advances in computer technology, much of these advances focused on the nuclear weapon's program. Similarly derived benefits for peaceful nuclear power generation have been taken in regard to modeling and simulation, particularly in the area of radiation transport. Indeed through the mid1980s the nuclear power industry was among the leading industries in utilizing the then leadership class scientific computing capabilities for commercial applications. With the downturn in nuclear power generation development in the USA and other countries, and the increasing power of the desktop workstation that occurred in the mid1980s, the nuclear power industry turned to porting their application software to workstations and to a large extent abandoned utilizing the leading edge high performance computing machines. This is not to say that there were not continued advances in modeling and simulation, but those advances were now constrained to a large extent to the workstation versus high performance computer capabilities. During this time, several other industries continued the adaption of high performance computing capabilities into their modeling and simulation development activities in support of design work. Most relevant for the nuclear power industry was the continued advancements in modeling and simulation built upon using high performance computing capabilities that was taking place in the nuclear weapon's complex, in particular the Accelerated Strategic Computing Initiative (ASCI) and subsequent Advanced Simulation and Computing (ASC) programs [1]. These programs were initiated at the time a ban on underground testing of nuclear weapons became USA policy, and took on the challenging task of modeling and simulating the multi-physics behavior of such weapons.

What high performance computing capabilities has 
enabled is the development of science-based, predictive capability. That is by the utilization of more science-based modeling, one expects to be able to apply predictive capability with a higher degree of confidence over a wider range of conditions. High performance computing along with advances in applied mathematics has also enabled the assembly into an integrated package a large collection of different physics modeling capabilities with tight coupling allowing the simulation of complex system phenomena involving multi-physics behaviors.

In this article, Section 2 discusses the multi-physics and multi-scale that are encountered in one aspect of nuclear power plant simulation and modeling, that being the modeling of the nuclear core. Section 3 summarizes the advances that have been made in high performance computing and the generic software that has been developed that exploits the capabilities of these computers. Section 4 presents examples of physics modeling and simulation performance on high end computers. In Section 5 conclusions are presented and some insights into the path forward are presented.

\section{MULTI-PHYSICS AND MULTI-SCALE}

A nuclear power station is composed of a collection of interacting systems. Many of these systems are complex and involve a range of physical phenomena. One of the more complex systems is the reactor core, where now radiation transport plays a important role. Fig. 1.a illustrates how most often today a reactor core simulation tool is constructed. The arrows displayed in Fig. 1 indicate the flow of information, so an arrow with only a one arrow tip indicates a one-way flow of information whereas an arrow with two arrow tips indicates two-way flow of information, i.e. feedback. What is striking in Fig. 1.a is the dominant one-way flow of information for the fuel performance simulator. This implies attributes such as thermal conductivity, gap closure, and fission product gas release are generally determined assuming some prescribed normal plant operating conditions, e.g. power history, with the resulting fuel properties communicated to other simulator components requiring them. By contrast, Fig. 1.b indicates a tighter integration with regard to coupling of many physics components. This can be done using Matrix Free Newton-Krylov (JFNK) (see [2] review article) or some alternative (see [3] for some alternatives for time-dependent problems), minimizing the need to employ operator splitting type mathematical coupling to achieve two-way coupling. When there truly is feedback involved between two or more physics simulators, the usage of one-way coupling will generally necessitate the introduction of conservatism, implying the need for design and safety margins greater than actually required leading to increased energy cost. With the usage of tighter twoway coupling, issues such as mathematical convergence of the simulation and introduction or removal of simulation predicted physical behaviors that are not real can be minimized.

How a multi-physics simulation capability should be built can vary. One approach would be to incorporate all the physics within a single code where programming calls to procedures are employed. That is how many core simulators, which contain both radiation transport and thermal-hydraulics simulation capabilities, and fuel performance simulators, which contain a host of physics models, are commonly constructed. A very different approach would be to develop the individual physics simulation capabilities and common support services separately, and then provide a software tool capable of integrating the separate pieces. Fig. 2 pictorially presents by example such an approach, the example being the Virtual Environment for Reactor Applications (VERA) simulator being developed by the Consortium for Advanced Simulation of Light Water Reactors (CASL). Similar activities are underway elsewhere, such as the NURESIM and follow up projects. Example of software integrators include LIME [4] used for VERA and SALOME [5] used by the NUclear REactors SIMulation (NURESIM) Integrated Project. In the center of Fig. 2 reside mathematical solvers and non-intrusive type sensitivity analysis/uncertainty quantification capabilities. By separating solvers, such as PETSc [6] and TRULENOS [7], from the physics routines, advantage can be taken of current

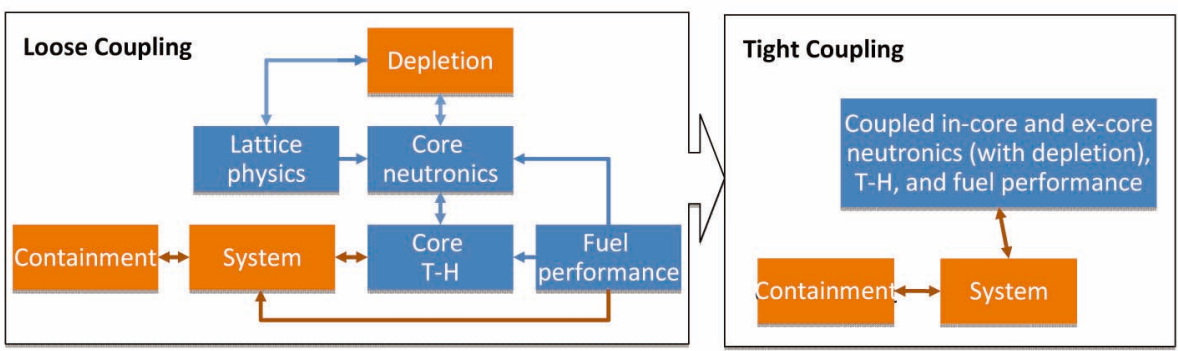

Fig. 1. Physics and Information Flow for a Nuclear Power Plant Simulator (a) with Loose Coupling and (b) with Tighter Coupling of Physics. 
and future generic advances made by others. The downside is that limitations can be introduced in taking full advantage of some special attribute of the equations being solved.

In the center of Fig. 2 is where non-intrusive sensitivity analysis/uncertainty quantification capabilities based upon statistical sampling [8] reside. By non-intrusive is implied the capability to complete sensitivity analysis/uncertainty quantification, with reference to input parameters, by only modifying the physics simulator's input and processing its output. Having such capabilities is becoming necessary when completing nuclear power plant simulations in order to make informed decisions, such as in accident analysis when utilizing the Best Estimate plus Uncertainty approach. However, non-intrusive approaches via statistical sampling, though straight forward to implement, may carry a heavy computational load burden. This computation burden can be reduced by employment of surrogate models, an example of which for uncertainty quantification and data assimilation can be found in treating nuclear data uncertainties in Ref [9]. By contrast intrusive approaches, such as the Adjoint Sensitivity Analysis Procedure (ASAP)[10,11], when

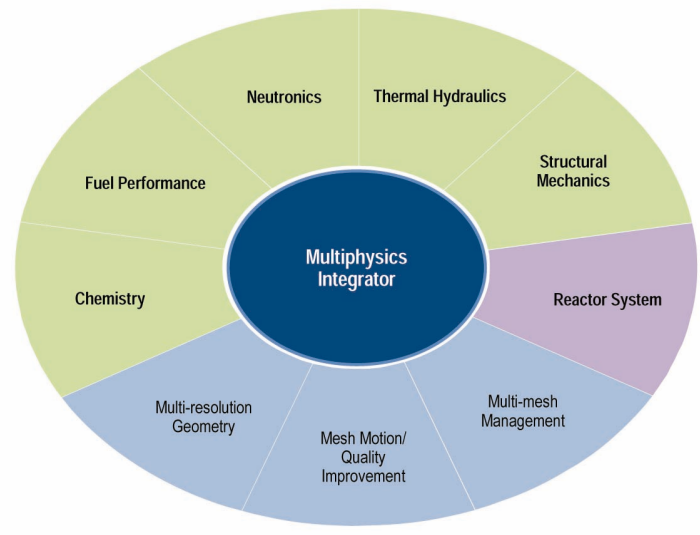

Fig. 2. Possible Organization of a Multi-physics Core Simulator. applicable, can be computationally efficient but can take considerable effort to implement in existing simulation capability.

Mesh support services depicted in Fig. 2 indicate all the different sort of meshing capabilities that are required. Mesh generation for areas of physics such as fluids and structural mechanics, can require a considerable computational load, complicated when utilizing Adaptive Mesh Refinement (AMR) [12]. Since each physics simulation has a preferred meshing, mesh support services must provide the capability of mapping results from one physics simulation to another physics simulation that requires those results. One certainly would not want to utilize the same mesh for radiation transport as for CFD. Further, for uncertainty quantification, we now need to not only know the numerical method introduced error uncertainty but also the additional error uncertainty that may be introduced by any mesh mapping operation. This is one example of multi-physics integration involving issues beyond the mathematical approach to integration.

Another area where the integration of simulation capabilities arises is in integrating the same physics but assessed at different scales. In developing science based, predictive capability, multi-scale simulation capabilities are necessary since computational power does not currently support simulations over the spatial and/or time domains of interest at the finest scales where the physics can be modeled in a more fundamental fashion. The motivations for developing multi-scale modeling and simulation capability are to have a deeper understanding of the mechanisms driving behavior at the engineering scale, and to have higher confidence in predictions at the engineering scale when outside the range of validation data. Examples of multi-scale applications can be found in fluids, where upscaling through the sequence of resolutions, e.g. Direct Numerical Simulation (DNS), Large Eddy Simulation (LES), Reynolds-Averaged Navier-Stokes (RANS) and subchannel, can be exploited, as depicted in Fig. 3. Likewise, upscaling is now more common in materials simulations again involving a sequence of resolutions, e.g. Density Functional Theory (DFT), Molecular Dynamics (MD), Phase Field,

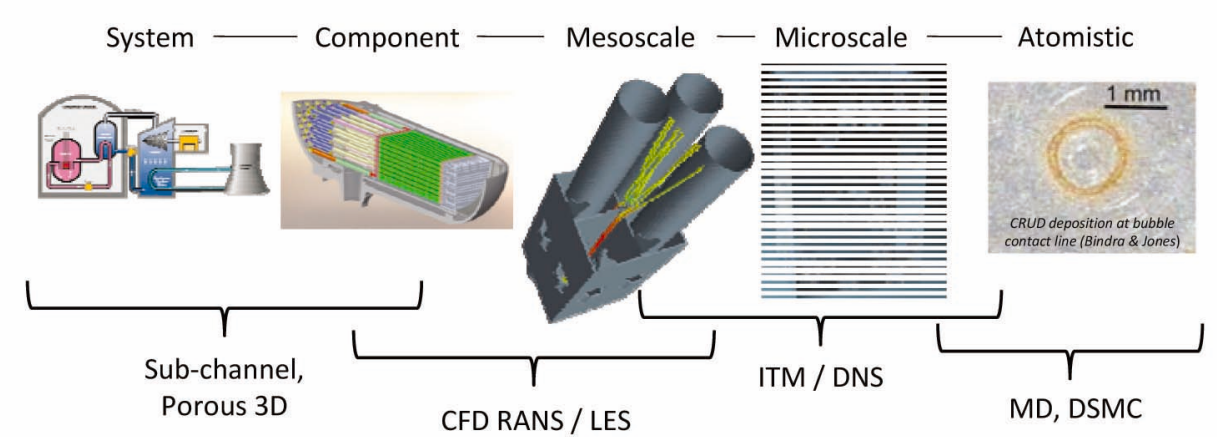

Fig. 3. Pictorial Representation of a Multi-level Approach to Fluid Dynamics Modeling and Simulation. 
and engineering continuum scale, as depicted in Fig. 4. In contrast to multi-physics coupling which has a unified mathematical basis for accomplishing, multi-scale coupling today remains very domain physics dependent. Differences also exist whether finer scales are employed to obtain closure relationships that are precalculated or the interactions between scales is dynamic. Fig. 5 shows an example [13] of the success in fluids of upscaling DNS to RANS with regard to matching mean velocity, turbulent kinetic energy, and turbulent dissipation.

Clearly the implementation of multi-physics and multiscale simulation capabilities to enable science-based, predictive capability will require considerable computational power. Also requiring enhanced computational power are sensitivity analysis, uncertainty quantification, and data assimilation. As noted earlier, licensing authorities have now accepted Best Estimate + Uncertainty as an acceptable basis for making licensing decisions. These decisions in most cases are still based upon limits on a collection of single attributes, such as pressure, temperature, stress, and reaction limits, serving as surrogates for failure of a structure, system or component (SSC). Given advances in modeling and simulation, removing the surrogate and actually predicting the failure of an SSC is now becoming possible. So what one would desire is a prediction of the probability of an SSC failing, which would further increase the computational power required. Sensitivity analysis and uncertainty quantification also are most useful in deciding on where future R\&D efforts should be focused. It is not beneficial to reduce the uncertainty contribution from a specific physics model if the system's attribute of interest uncertainty is dominated by uncertainties in other physics models. Likewise, unwise utilization of resources would result if the major contributor to the uncertainties
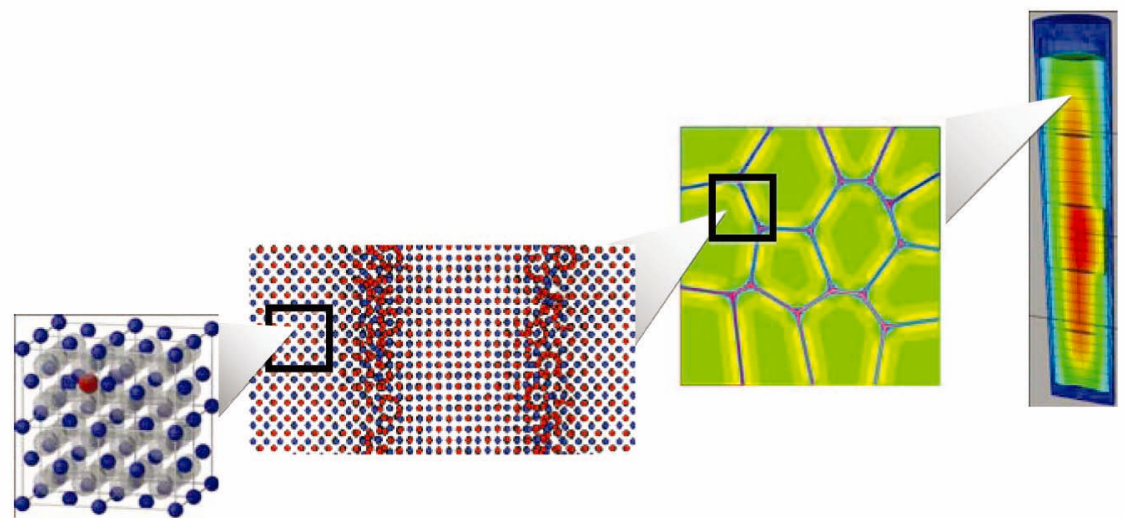

Fig. 4. Pictorial Representation of a Multi-level Approach to Materials Modeling and Simulation.
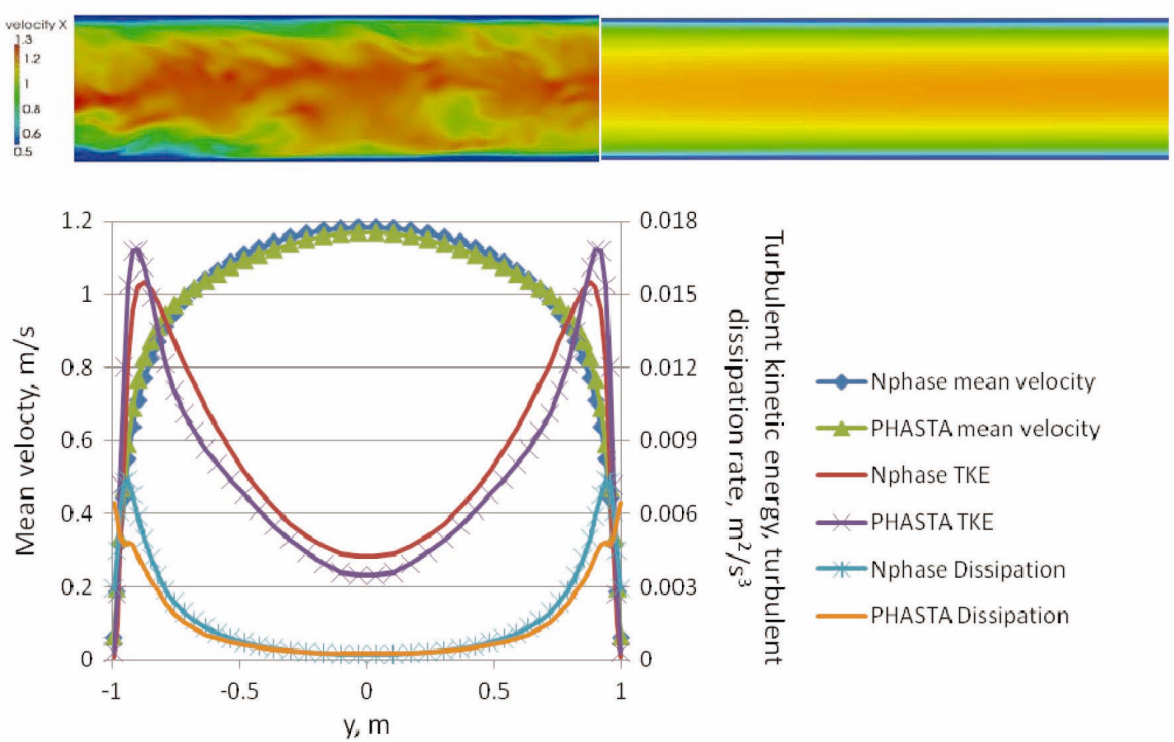

Fig. 5. Effectiveness of Up-scaling DNS (PHASTA) Results for RANS (NPHASE) Simulation of Multi-phase Flow. 
of interest for a specific physics simulation originates overwhelmingly due to parameters, i.e. code input and closure relationships, but the effort is being expended on improved modeling. Another example where significant computational power is required is executing simulations that integrate PRA with system simulations, enabling a broader assessment of possible accident sequences. The Risk-Informed Safety Margin Characterization (RISMC) work being done at Idaho National Laboratory [14] is an example of an effort to develop such a capability.

What is clear from the above is that computational power at the leading edge will be required to develop and utilize advances in modeling and simulation for nuclear power stations. If successful, this will enable a deeper understanding of the phenomena involved and associated uncertainties of complex engineering systems, which in turn will produce the following benefits:

- superior product designs,

- reduced product development cycle time,

- reduced product development cost due to time and experimental cost savings,

. enlarged operating space, e.g. power uprates, and

- more robust safety analysis supporting the licensing case.

\section{HIGH PERFORMANCE COMPUTING}

High performance computation power continues to increase at a rapid rate. As measured by performance on some benchmark problems, the mean lifetime of holding the position as the fastest computer in the world is more appropriately measured in months versus years. Architectures of the machines have evolved with time, both with regard to CPU and memory organization, impacting interconnection architecture. Within recent times three hardware features have most significantly contributed to increasing the performance on scientific problems, those being massive parallelization of processor nodes, increasing number of cores per node, and most recently utilization of accelerators, e.g. GPUs [15]. Over the last decade the performance increase achieved by parallelization versus processor speed as measured by FLOPS has been approximately 25 times greater. Supporting this increase in computational power are enhanced internal networks, memories, and I/O devices. Fig. 6 presents the performance of the leading computers of the time versus year [16].
For the current trend in rate of performance increase to continue, some fundamental issues will need to be addressed. High on the list is the development of higher performing processor nodes but with reduced energy consumption required. Processor nodes will need to have many more threads, and each thread will need to support many more vectors, providing a hierarchy approach to achieve massive parallelization. In addition, enlarged hierarchal memories and better interconnections will be needed to support the massive parallelism. If predictions are correct, performance over this decade will increase from a few petaflops to exaflops, allowing problems that now take 24 hours to execute to be completed in about 1.5 minute provided the computational power can be exploited in application software. To achieve this performance, programming models will need to change to take advantage of the massive hierarchal architecture. Indeed it may prove more computationally desirable to utilize a particle based model versus a partial differential equation based model to represent some physics.

For the nuclear power industry to supplement desktop computers with higher performance machines, the cost must be justified by the economic benefits to be derived. Expected benefits were listed at the conclusion of Section 2. Regarding computational cost, Table 1 [17] indicates the cost per GFLOPS as a function of time. What Table 1 indicates is that the performance of today's high performance

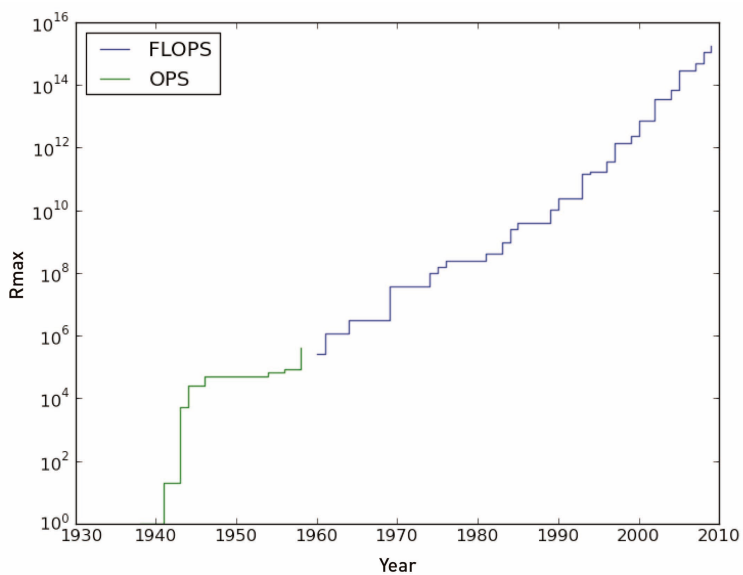

Fig. 6. Time History of Floating Point Arithmetic Performance of Leading Edge Computers.

Table 1. Time History of Processor Cost Per Unit GFLOPS of Performance.

\begin{tabular}{c|c|c|c|c|c|c|c}
\hline Date & 1961 & 1984 & 1997 & 2000 & 2003 & 2007 & 2011 \\
\hline Cost/GFLOPS & $\$ 1.1$ trillion & $\$ 15$ million & $\$ 30,000$ & $\$ 640$ & $\$ 82$ & $\$ 48$ & $\$ 1.80$ \\
\hline
\end{tabular}


machines will be realizable in the not too distant future at a modest price. Indeed that is the reason why advances in modeling and simulation over the last two decades have been possible employing ever more powerful desktop computers. However, as noted above, parallelization has increased computational power 25 times more than processor speed, implying that to support the advances in modeling and simulation noted in Section 2, the usage of machines with more parallel architectures at all levels will be the quickest path to obtaining the capabilities required. This in turn will require different programming models to properly exploit the parallelism of the hardware.

\section{HIGH PERFORMANCE COMPUTING APPLICATIONS}

Focusing on the core simulator as depicted in Fig. 2, it is of interest to review the modeling and simulation capabilities of physics problems that can be addressed currently, and the computational time to solve these problems as a function of degree of parallelization. Radiation transport simulation is a key component of a core simulator, since in addition to predicted quantities directly interpreted, e.g. power peaking factor, it provides information utilized by the thermal-hydraulic and fuel performance simulators. For the thermal-hydraulic simulator, the power density throughout the core is obtained from the radiation transport simulator; whereas, for the fuel performance simulator, the elemental concentrations and interaction rates are obtained from the radiation transport simulator. Today radiation transport simulations are completed by the nuclear industry utilizing lattice physics codes to calculate using transport theory the few-group, homogenized cross-sections, discontinuity factors, and form factors needed for pinpower reconstruction. This is followed by nodal based solution of the few-group, neutron diffusion theory equations over the entire core. This approach is based upon a sequence involving energy group collapsing and spatial homogenization, proven very successful for current LWRs with LEU cores for the intended utilization of the core responses predicted. Various approximations are utilized to decouple the transport theory based, lattice physics calculations from the diffusion theory based, core-wide calculation, most notable the assumption of reflective boundary conditions for the lattice geometry. Collapsing to few-groups, spatial homogenization, and making the diffusion theory approximation renders it not possible to correct this boundary condition assumption in a mathematically rigorous fashion. Also, to complete higher fidelity thermal-hydraulic and fuel performance simulations, pin-resolved detail including power density and isotopic interactions are required, which is not available from current pin reconstruction algorithms. Hence if either higher fidelity over the prevalent current methods or pin resolved detail is required, one needs to consider on a

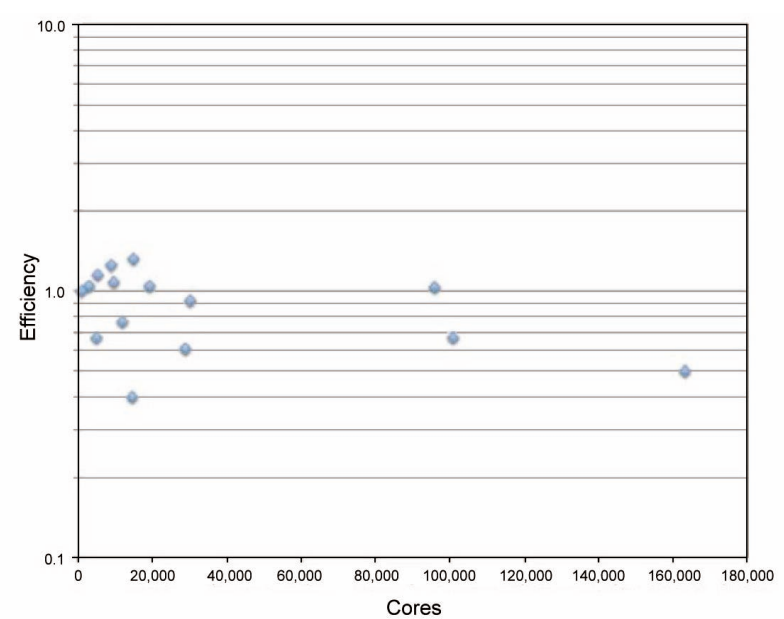

Fig. 7.Computational Parallel Efficiency Performance of the DENOVO Radiation Transport Code for Weak Scaling.

whole core basis the utilization of many-group, spatially resolved deterministic transport or stochastic Monte Carlo calculations. The degrees of freedom will be very large for such calculations.

Fig. 7 presents results from the Oak Ridge National Laboratory developed DENOVO code [18], an SN transport code, using cell-homogenized cross sections with stepcharacteristics, linear-discontinuous finite elements and trilinear-discontinuous finite element spatial methods. The figure shows weak scaling results for a PWR core using 44 energy groups, and a range of number of spatial nodes and angular discretizations, producing up to 1.7 trillion degrees of freedom. This code was executed on the ORNL Jaguar XT5 computer, which is rated at about 2 petaflops, utilizing up to 160,000 cores. Excellent parallel scaling is noted in Fig. 7. The non-smoothness of results originates due to non-proportional increases in the number of spatial and angular degrees of freedom used in the weak scaling study, since parallel performance is different for the spatial and angular domains. Note that even with the computational power of Jaguar, to reduce the execution time to what a core designer experiences today running a nodal diffusion code on a desktop computer would imply the need to employ an exascale rated computer. Calculations as reported here will in the near term be restricted to applications such as establishing computational benchmarks, completing generic design and safety analysis studies, and addressing unique plant specific problems. An open question does exist regarding what level of accuracy is needed of the radiation transport simulator given the uncertainties in the thermal-hydraulic and material simulators that will utilize the former simulator's predictions, and for the radiation transport simulator what contribution does modeling error uncertainties contribute to the overall simulator's uncertainties. 

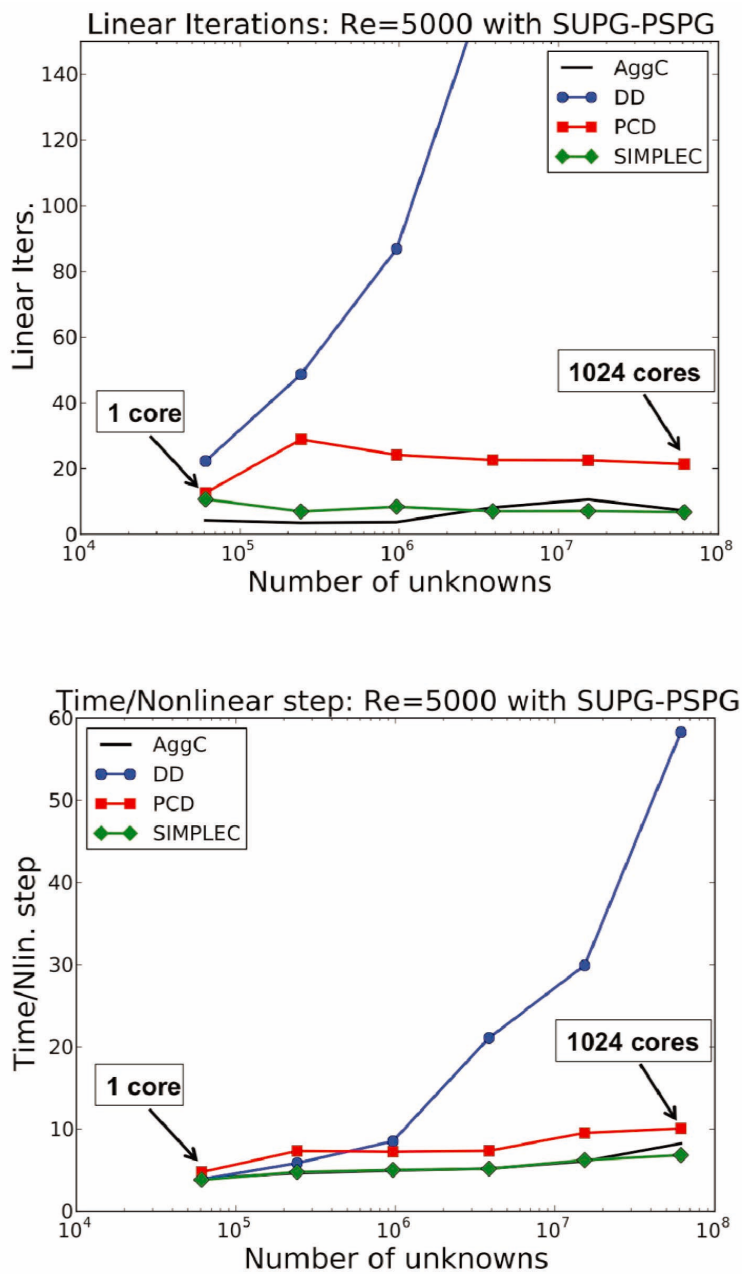

Fig. 8. Computational Performance of the Charon Code Applied to Fluid Dynamics for Various Preconditioners for Weak Scaling.

For thermal-hydraulic modeling and simulation, the trend has been to attempt to complete detailed core simulations utilizing CFD, particularly for single-phase flow conditions where modeling capabilities are more mature versus for two-phase flow conditions. CFD provides the potential to predict more accurately fluid flow rates, pressures and temperature, and with cognizant heat transfer fuel and cladding temperatures, on a fine spatial scale. It also provides the time dependent pressure field due to turbulence acting on the fuel cladding required by structural mechanics models to predict fuel rod vibration and hence grid-to-rod-fretting (GTRF). Several commercial CFD codes have been utilized for nuclear power applications. Coupling of CFD using RANS and radiation transport simulators via operator splitting has already been demonstrated [19] for core-wide LWR calculations, based upon what would be considered a coarse CFD grid. Fig. 8 presents performance results for the Charon code [20] developed at Sandia National Laboratory being applied to solve the incompressible Navier-Stokes

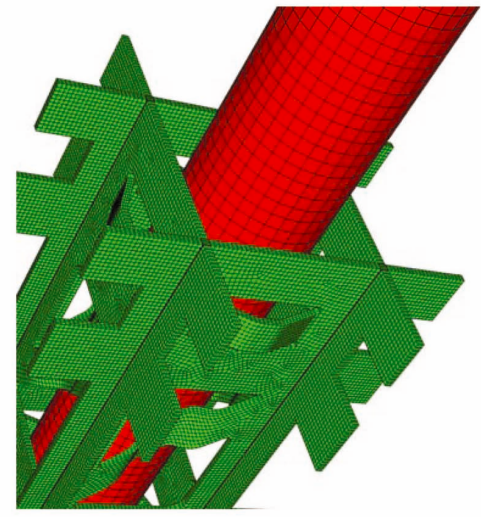

Rod: 75008 Hex8 Elements

Grid: 94536 Hex8 Elements in each of 3 grids. 3 elements through thickness of plates

Fig. 9. Representative Spatial Grid Meshing Utilized to Complete the Structural Analysis for GTRF.

fluid equations. The specific application being analyzed is a Kelvin-Helmholtz shear layer. Briefly, as being used here, Charon provides a second-order accurate solution in space employing unstructured stabilized finite element method and $1^{\text {st }}$ to $5^{\text {th }}$ order fully-implicit time treatment. Using a Newton-Krylov method, Fig. 8 shows for various Krylov preconditioners (see Ref. [20]) performance as measured regarding the number of Krylov iterations and Wall Clock Time per Newton step, with computational results for weak scaling on the Sandia Red Sky computer. A derivative of Charon, Drekar, has been used to obtain the turbulent pressure forces used in GTRF analysis.

Other aspects of completing a GTRF analysis include structural mechanics and fuel performance. In Fig. 9 is a depiction of the meshing that was used to complete the structural analysis, with verification of this grid remaining to be completed to assure adequate fidelity. Fig.10 presents for a three grid span the forces acting on the fuel rod due to vibrational contact with the spacer grid, assuming for the top and bottom grids firm contact and for the middle grid an initial gap between the fuel rod and spacer grid caused by spacer grid growth and initial fuel rod radial growth following by shrinkage [21]. Earlier times are used to establish the top and bottom grids contact forces. Drekar using LES was employed to determine the turbulent forces and Adagio, a quasi-static structural mechanics module which is part of the Sandia National Laboratory Sierra [22] computational framework, was employed to determine the fuel rod vibration. The Adagio code module employs a Lagrangian, three-dimensional, implicit method for the analysis of solids and structures. Fig. 11 shows weak scaling results for Adagio, which indicates the challenge of achieving high parallel efficiencies for structural analysis codes. For ideal weak scaling, clock time should not increase as the number of processors is increased. However, factors such as non-parallizable (serial) fraction of code, communications overhead, and total floating point operations (FLOP) increase as number of processors 


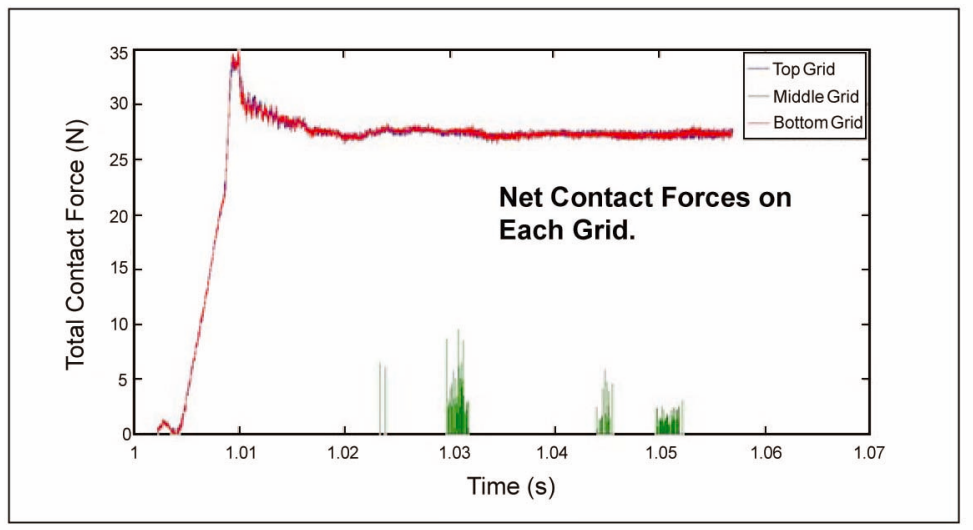

Fig. 10. Contact Forces between Spacer Grids and Fuel Rod Leading to GTRF.

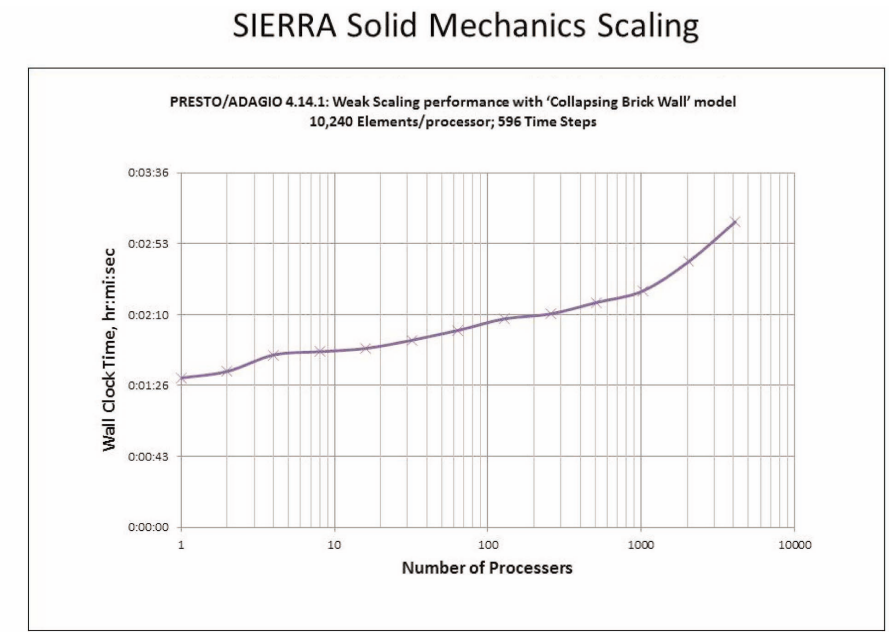

Fig. 11. Computational Parallel Efficiency Performance of the Sierra-Adagio Structural Analysis Code for Weak Scaling

increase due to introducing a parallizable solution algorithm, e.g. domain decomposition, can lead to less than ideal weak scaling.

The final example comes from a fuel performance simulation. In contrast to the other predominantly single or few physics simulators discussed, fuel performance simulators on their own are multi-physics simulators, containing heat transfer, fluid, structural mechanics, chemistry, and materials physics models. These models can range from closure relationships, e.g. thermal conductivity as a function of material temperature and fluence, to engineering scale mathematical models, e.g. partial differential equation for heat transfer. Fig. 12 presents weak and strong scaling results for the BISON fuel performance model developed by Idaho National Laboratory, one of the models developed under the MOOSE computational framework [23]. A fully implicit time integration and fully-coupled multi-physics framework is employed. The problem being analyzed consists of 320 pellets with 234 million degrees of freedom, and represents heat transfer and structural mechanics in three dimensions. The figure shows good scaling on a high performance computing platforms using 11,820 processor cores on INL's Fission high performance computer.

\section{SUMMARY}

What has been presented is a brief introduction to the opportunities and challenges of an increased utilization of high performance computing for nuclear power plant applications. Considerable resources and time will be required to take full advantage of the computational power offered by high performance computing. Moving towards 


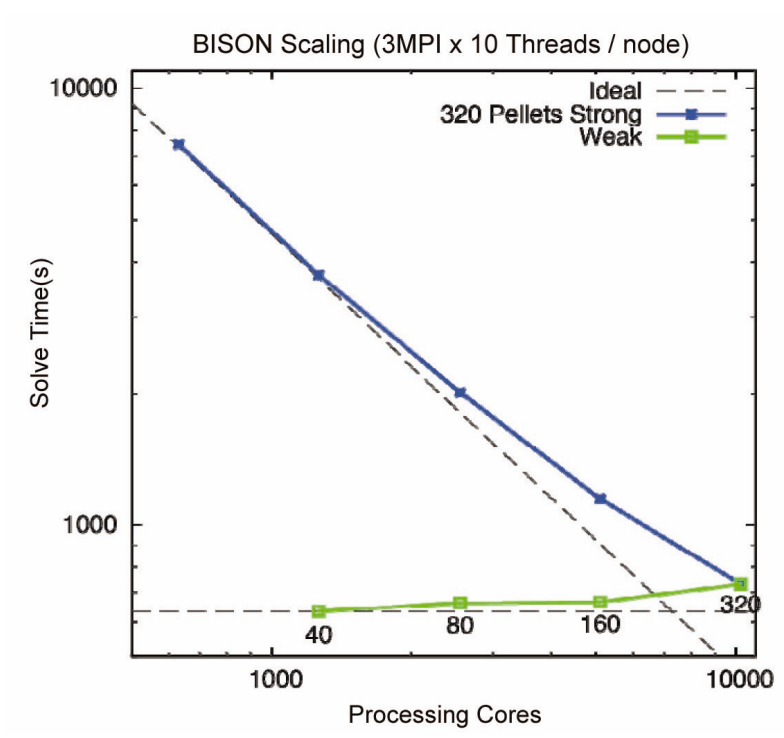

Fig. 12. Computational Parallel Efficiency Performance of the MOOSE-BISON Fuel Performance Code for Weak and Strong Scaling.

science based, predictive capability for a single area of physics is challenging due to multi-scale phenomena; however, the development of predictive nuclear power plant simulations is a much greater challenge due to the need to also accurately represent multi-physics. Significant progress is beginning to be made, with modeling and simulation capabilities developed being applied to better understand the behavior of nuclear power plants, which in time will lead to improved designs and a heightened assurance of nuclear safety. Given the significant efforts in validation and regulatory licensing of existing simulation capabilities, one can expect a slow transition to utilizing science based, predictive simulation capabilities made possible utilizing high performance computers even if the economic case for doing so is strong.

\section{ACKNOWLEDGEMENT}

The author would like to thank individuals involved with the Consortium for Advanced Simulation of Light Water Reactors, a US Department of Energy Innovation Hub, for providing a number of the figures that are contained in this article.

\section{REFERENCES}

[1] NNSA Advanced Simulation \& Computing, Home Page, http://www.nnsa.energy.gov/asc.

[2 ] D.A. Knoll and D.E. Keyes, "Jacobian-free Newton-Krylov methods: a survey of approaches and applications," Journal of Computational Physics, vol. 193, pp. 357-397 (2004).

[3] Robert B. Lowrie, "A Comparison of Implicit Time Integration Methods for Nonlinear Relaxation and Diffusion," Journal of Computational Physics, vol. 196, issue 2, pp. 566-590 (2004).
[4 ] Roger Pawlowski, Roscoe Bartlett, Noel Belcourt, Russell Hooper, and Rod Schmidt, "A Theory Manual for Multiphysics Code Coupling in LIME - Version 1.0," SAND20112195, Sandia National Laboratory (2011).

[5] SOLOME: The Open Source Integration Platform for Numerical Simulation, Home Page, http://www.salomeplatform.org/.

[6] PETSc: Portable, Extensible Toolkit for Scientific Computation, Home Page, http://www.mcs.anl.gov/petsc/ index.html.

[ 7 ] Trilinos, Home Page, http://trilinos.sandia.gov/.

[ 8 ] Brian M. Adams, Keith R. Dalbey, Michael S. Eldred, Laura P. Swiler, William J. Bohnhoff, John P. Eddy, Dena M. Vigil, Patricia D. Hough, and Sophia Lefantzi, "DAKOTA, A Multilevel Parallel Object-Oriented Framework for Design Optimization, Parameter Estimation, Uncertainty Quantification, and Sensitivity Analysis, Version 5.2+ Theory Manual," SAND2011-9106, Sandia National Laboratory (2011).

[9] M. A. Jessee, P. J. Turinsky, and H. S. Abdel-Khalik, "Many-Group Cross-Section Adjustment Techniques for Boiling Water Reactor Adaptive Simulation," Nuclear Science and Engineering, vol. 169, no. 1, pp. 40-55 (2011).

[10] Dan G. Cacuci, Sensitivity and Uncertainty Analysis: Theory, vol. 1, Chapman \& Hall (2003).

[11] Dan G. Cacuci, Mihaela Ionescu-Bujor, and Michael Navon, Sensitivity And Uncertainty Analysis: Applications to LargeScale Systems, vol. 2, Chapman \& Hall (2005).

[12] Tomasz Plewa, Timur Linde, and V. Gregory Weirs, (Eds.), "Adaptive Mesh Refinement - Theory and Applications," Proceedings of the Chicago Workshop on Adaptive Mesh Refinement Methods, Sept. 3-5, 2003, Series: Lecture Notes in Computational Science and Engineering, vol. 41, Springer (2005).

[13] I.A. Bolotnov, S.P. Antal, K.E. Jansen and M.Z. Podowski, "Multidimensional Analysis of Fission Gas Discharge following Fuel Element Failure in Sodium Fast Reactor," 13th International Topical Meeting on Nuclear Reactor Thermal Hydraulics (NURETH-13), Kanazawa City, Ishikawa Prefecture, Japan (2009).

[14] RISMC: Risk Informed Safety Margin Code, https:// inlportal.inl.gov/portal/server.pt/community/lwrs_program /442/rism_characterization_continued.

[15] Wikipedia,http://en.wikipedia.org/wiki/GPGPU.

[16] Wikipedia, http://en.wikipedia.org/wiki/Supercomputer.

[17] Wikipedia, http://en.wikipedia.org/wiki/FLOPS.

[18] Joshua J. Jarrell, Andrew T. Godfrey, Thomas M. Evans, and Gregory G. Davidson, "Full Core Reactor Analysis: Running Denovo on Jaguar," PHYSOR 2012 Advances in Reactor Physics Linking Research, Industry, and Education, Knoxville, TN, USA (2012).

[19] David P. Weber, T. Sofu, P. A. Pfeiffer, W. S. Yang, T. A. Taiwo, H. G. Joo, J. Y. Cho, K. S. Kim, T. H. Chun, T. J. Downar, J. W. Thomas, Z. Zhong, C. H. Kim, B. S. Han, "The Numerical Nuclear Reactor for High Fidelity Integrated Simulation of Neutronic, Thermal-Hydraulic and Thermo Mechanical Phenomena - Project Overview," PHYSOR 2004 - The Physics of Fuel Cycles and Advanced Nuclear Systems: Global Development, Chicago, IL, USA (2004).

[20] Eric C. Cyr, John N. Shadid, and Raymond S. Tuminaro, 
"Stabilization and scalable block preconditioning for the Navier-Stokes equations," Journal of Computational Physics, vol. 231, pp. 345-363 (2012).

[21] Nathan Crane, Rick Garcia, Tim Shelton and Kendall Pierson of SNL, and Sam Sham and Ramanan Sankaran of ORNL, Private Communication (2011).

[22] James R. Stewart and H. Carter Edwards, "The SIERRA Framework for Developing Advanced Parallel Mechanics Applications," in Large-Scale PDE-Constrained Optimization,
Lecture Notes in Computational Science and Engineering, Editors: Lorenz T. Biegler, Omar Ghattas, Matthias Heinkenschloss, and Bart van Bloemen Waanders vol. 30, part VII, pp. 301-315 (2003).

[23] Derek Ray Gaston, Glen Hansen and Christopher Newman, "MOOSE: A Parallel Computational Framework for Coupled Systems of Nonlinear Equations," International Conference on Mathematics, Computational Methods \& Reactor Physics, Saratoga Springs, NY, USA (2009). 